Research Article

\title{
Decision-Making Method of Qualitative and Quantitative Comprehensive Evaluation of Talents Based on Probability Hesitation Fuzzy Language
}

\author{
Xueer Ji $\mathbb{D}^{1},{ }^{1}$ Lei Wang $(\mathbb{D})^{2}$ Huifeng Xue $\mathbb{D}^{1},{ }^{1}$ and Yufeng Gao ${ }^{1}{ }^{1}$ \\ ${ }^{1}$ China Aerospace Academy of Systems Science and Engineering, Beijing 100035, China \\ ${ }^{2}$ School of Economics and Management, Xi'an University of Posts and Telecommunications, Xi'an 710065, China \\ Correspondence should be addressed to Xueer Ji; jixueer@mail.nwpu.edu.cn
}

Received 22 July 2021; Revised 26 October 2021; Accepted 24 November 2021; Published 21 December 2021

Academic Editor: Musavarah Sarwar

Copyright (C) 2021 Xueer Ji et al. This is an open access article distributed under the Creative Commons Attribution License, which permits unrestricted use, distribution, and reproduction in any medium, provided the original work is properly cited.

A scientific, reasonable, and novel talent evaluation index system is the foundation of talent training and selection. Based on the novel "Man-Machine-Environment System Engineering" (hereinafter referred to as MMESE) theory, this paper proposes a novel talent evaluation index system that considers the ontological attributes and the external environment of the object comprehensively for talent evaluation, which could help the evaluator obtain more accurate evaluation results. Since the comprehensive evaluation of MMESE talents is a complex decision-making problem that is both qualitative and quantitative, a corresponding decision-making method that integrates qualitative and quantitative approaches is proposed here based on probabilistic language entropy and the possibility of superior order relationships. First, the weights of quantitative and qualitative attributes are calculated based on entropy theory and probabilistic fuzzy language. Second, the standard weight vectors of qualitative and quantitative attributes are obtained by adjusting the weight integration coefficients, and the change intervals of the pros and cons between the objects to be evaluated are calculated. Third, the pros and cons of the objects to be evaluated are compared to obtain the possibility degree matrix that describes the priority relationships among the objects, and a ranking vector is derived from the possibility degree matrix to reflect the rankings of the objects' pros and cons. Finally, this system and the decision-making methods have been verified as scientific and effective.

\section{Introduction}

The world is experiencing a serious shortage of talents in the twenty-first century. Aside from the uneven economic development levels, the main reason for such a shortage is the lack of a scientific and comprehensive mechanism for talent training and selection. Therefore, the research on talent evaluation index systems and talent evaluation methods is of great significance to the formulation of strategic plans for talent training and the emergence of global talents. Particularly, a scientific and reasonable talent evaluation index system is the foundation of talent training and selection, and such a system has been studied extensively for the affecting factors. Tian et al. [1] focused on the connotation, characteristics, and growth rules of talents and studied the impact of the integration of external environments (e.g., social, group, cultural, educational, and natural) and internal factors (e.g., physiology, psychology, and intelligence) on talent growth. Qiu [2] built an evaluation system that is both qualitative and quantitative to evaluate college students for potential international applied talents in the context of internationalization. $\mathrm{Li}$ [3] improved the evaluation system for the education results of higher vocational students with a quality and ability evaluation index system specially designed for these students. Nan et al. [4] proposed an innovation-oriented talent evaluation system for greater achievements of the innovation-driven development strategy. From the perspective of the human resources value 
chain, Shang and Wang [5] created an evaluation index system for the talent ecosystem, in which three first-level indicators, namely, talent competence, organizational ability, and regional environment, are evaluated. These research findings have greatly promoted the fairness and righteousness of the talent evaluation and selection process.

As for the talent evaluation methods, studies have been focused on the competitive evaluation method, analytic hierarchy process, and fuzzy evaluation method, and so on. For example, Jia et al. [6] used the competitive evaluation method to design index weights and evaluation models for advanced talents in scientific and technological fields. Labi et al. [7] recommended using AHP in four evaluation steps when selecting employees. Metin and Deha Er [8] proposed a fuzzy comprehensive multistage multicriteria evaluation model for management and academic talents. Lazarevic [9] proposed a fuzzy two-level model to minimize subjective judgment in employee selection. Drigas et al. [10] used the neurofuzzy technique to investigate and evaluate unemployed people or those working in specific positions.

Talent evaluation involves various internal and external factors and complex component systems, especially under the context of rapid social and economic developments. Vague and uncertain information generated during the evaluation process is difficult to handle with the currently available quantitative evaluation models. In 1981, a comprehensive edge technology named Science-Man-MachineEnvironment System Engineering (SMMESE) [11] was born, which provided an important theoretical foundation to build talent evaluation index systems in complex environments. In 1990, based on SMMESE, Qian and his colleagues put forward the "integrated method from qualitative evaluation to quantitative evaluation" [12], which is effective for talent evaluation. The core of Qian's method is to organically integrate quantitative data and expert experience, knowledge, and subjective judgments. However, as the talent evaluation index system becomes more complex, decisionmaking experts are constrained by their knowledge structure and levels, making the quantification of complex qualitative indicators difficult.

To tackle such a problem, experts have begun to use language terms as the way to express information, such as Zadeh's [13] fuzzy language method proposed in 1975. In Zadeh's method, qualitative decisions are represented as language variables such as "good" or "bad," and this method enhances the feasibility and flexibility of decision expressions. Studies in numerous fields have testified the reliability of this method, and this method has been extensively studied for extensions into fuzzy decision-making methods. For example, Ali et al. [14] built an MADM decision model based on the q-rung orthopair fuzzy bipolar soft sets. Ali et al. [15] studied the fuzzy bipolar soft expert-based decision-making method and implemented it in a COVID-19 situation.

However, the fuzzy language method only uses single linguistic terms to give value to linguistic variables, which could be insufficient and inaccurate to reflect the real expert opinions. For this reason, Rodriguez et al. [16] proposed the concept of hesitant fuzzy linguistic term set (HFLTS), in which text-free grammars and conversion functions are used to transform the expert's decision languages into operable hesitant fuzzy language sets. Following that, HFLTS has been applied in various decision-making methods for language information. $\mathrm{Xu}$ and Gou [17] proposed the concepts of hesitation fuzzy entropy and hesitation fuzzy cross-entropy and applied them to multicriteria decision-making problems. HFLTS provides possible language terms for decisionmaking experts, and these language terms are assumed to have equivalent importance in decision-making. However, in actual decision-making problems, since all experts have their preferences, the importance of different language terms is often varied. Therefore, Pang et al. [18] extended HFLTS to probability linguistic term set (PLTS) to reflect such variations, which allows experts to assign probabilities for multiple linguistic terms. Wang and Xue [19] proposed a comprehensive decision-making method that incorporates quantitative data and linguistic probabilities based on a comprehensive integrated discussion method.

In summary, the available literature has provided many references for the construction of talent evaluation index systems and evaluation methods, but there are still several shortcomings. Firstly, a talent evaluation index system is primarily a systematic analysis of human factors and the external environment, but previous methods failed to consider the impact of "machines" on talent training in the information era. Secondly, the lack of scientificity in talent evaluation methods makes them difficult to perform comprehensive, quantitative, and qualitative evaluations in a complex environment. Thirdly, available evaluation models have found it difficult to accurately express the qualitative indicators in the talent indicator system, and therefore the fuzziness of these indicators is not represented in the evaluation results.

To solve these deficiencies, the internal and external factors affecting talent growth are comprehensively analyzed based on the theory of MMESE in this paper. Following that, an MMESE talent evaluation index system is created to comprehensively expand the connotation of the talent evaluation index system. In addition, the theories of comprehensive integration methods, probabilistic language fuzzy entropy, and superior order relationship are employed in this paper for the comprehensive evaluation of fuzzy quantitative and qualitative indicators in talent evaluation. Finally, a qualitative and quantitative indicator is proposed to the MMESE talent evaluation index system to verify its effectiveness and scientificity.

\section{The MMESE Talent Evaluation Index System}

2.1. Theory of Construction. In the big data era, external devices have brought us massive information and changed the ways people use to acquire knowledge. Biology and machines tend to integrate into the new era, and the cuttingedge brain-computer interface technology has become a strategic hot spot in many countries. The contradiction between the explosive growth of human knowledge and the slow development of biological brains has become more prominent than it has ever been. Moreover, the growth models of talents across times and regions change with the 
local informatization level, resulting in more significant limitations of the traditional talent evaluation index system. Therefore, a scientific and rational talent evaluation index system for the big data era is needed to address the influences of machine development, as well as economic, political, social network, and other external factors, on talent growth.

Particularly, MMESE believes that the interactions among man, machine, and environment make up not a simple cycle but a spiral growth process [20]. Electronic computers have shifted the role of humans from a direct operator to a decision-maker, leading to significant enhancement, partial replacement, and great expansions of the functions of human mental work. In this way, human beings are assigned to more advanced and smart activities. MMESE theory is an important foundation of talent evaluation indicators. Besides, within the vigorous development of science and technology, almost all cutting-edge scientific research and technological work are controlled by computers. Consequently, talent emergence is experiencing a boom, and traditional talent evaluation index systems need corresponding changes.

In this paper, the coupling relationships between human and machine, human and environment, and machine and environment are analyzed under three complex systems. Additionally, an MMESE talent evaluation index system that involves both qualitative and quantitative factors is proposed. Particularly, MMESE does not merely study the performance of human, machine, and environment; it also involves special considerations about the coupling relationship between the three elements and the methods to coordinate them to build an MMSES talent evaluation index system. There are seven aspects in the MMESE theory (Figure 1).

2.2. Factor Analysis of the MMESE Talent Evaluation Index System. The talent evaluation index system in the MMESE theory is a dynamic, open, and multilevel model involving both qualitative and quantitative indicators. Specifically, personal ability, physical and mental health, moral quality, family environment, social environment, and innovation ability are some of the most-mentioned indicators [20-23]. Based on the available research results related to talent evaluation index elements, this paper combines the MMESE theory with these elements and divides the factors involved into three levels: human (B1), machine (B2), and environment (B3). When selecting the indices, the principles of pertinence, systematization, completeness, practicability, and foresight were strictly followed, and the indices selected in the first round were screened for better performance of the MMESE talent evaluation system.

2.2.1. Factors of the B1 Layer. In the B1 layer "Human," talents are evaluated from four aspects, namely, health level $(C 1)$, psychological quality $(C 2)$, education level $(C 3)$, and innovation ability $(C 4)$.

$C 1$ is focused on health risk assessments, and talents with relatively stable physical fitness are given good evaluations because they are considered to be able to engage in long-term, stable, and continuous growth. Since 1980, the life expectancy worldwide has increased by 10 years to 74.8 years in 2015 . However, the annual death toll has also risen from 48 million in 1990 to nearly 56 million in 2015. Specifically, chronic diseases are still one of the major health problems for humans. According to the 2016 World Health Organization (WHO) report, there are more than 6 billion subhealthy people in the world, accounting for $85 \%$ of the total population [24]. Unhealthy conditions can make people feel burnt out or unresponsive, leading to lower vitality and adaptability. Moreover, many people are frequently finding themselves in a state of work anxiety, irritability, and helplessness, and these conditions will bring serious undesired impacts to talent development. Therefore, the indicators in the $C 1$ aspect are used as quantitative parameters for personal health analysis. Health risk assessment [25] is a tool that describes and evaluates the possibility of an individual suffering or dying from a specific disease at a specific time in the future. It is indeed a quantitative assessment of the individual's health status and the risk of future illness and death for him/her.

The $C 2$ aspect is focused on mental health. WHO defines health to be more than the absence of disease and weakness. It also includes the physical, psychological, and social integrity of an individual. In 2001, WHO further defined mental health as a state of health or happiness in which individuals can realize themselves, cope with the pressures of daily life, work productively, and contribute to the society in which they live [26]. Some scholars believe that mental health is essential for an individual's overall health, and it is a continuous psychological state in which the individual shows good vitality, positive inner experience, satisfactory social adaptation, and effective production capability, thereby giving full play to his/her physical and mental potentials [27]. Therefore, the main evaluation criteria in $C 2$ are self-harmony, interpersonal harmony, and social harmony.

The C3 aspect addresses education. A person's knowledge level is reflected in his/her academic qualifications and knowledge structures. School education is designed to cultivate people with particular plans in designated institutions, and schools are the foundation of talent growth. Therefore, the $\mathrm{C} 3$ aspects consider the highest education level of the individual.

The $C 4$ aspect considers the individual's innovation ability. In 1950, JP Guilford (1950) delivered a famous speech entitled "Creativity" at the American Psychology Annual Conference, calling for joint research on creative issues. From then on, the research on the quality of innovative talents has flourished till now. Here in this paper, the innovation ability refers to the talents' ability to propose new ideas, theories, methods, and inventions with economic, social, or ecological value in practical activities.

With the analysis above, the detailed indicators of the "Human" layer in the MMESE talent evaluation index system are obtained (Table 1).

2.2.2. Factors of the B2 Layer. In the B2 layer "Machine," talents are evaluated from two aspects, namely, daily information downloads (C5) and computer application capabilities (C6). 


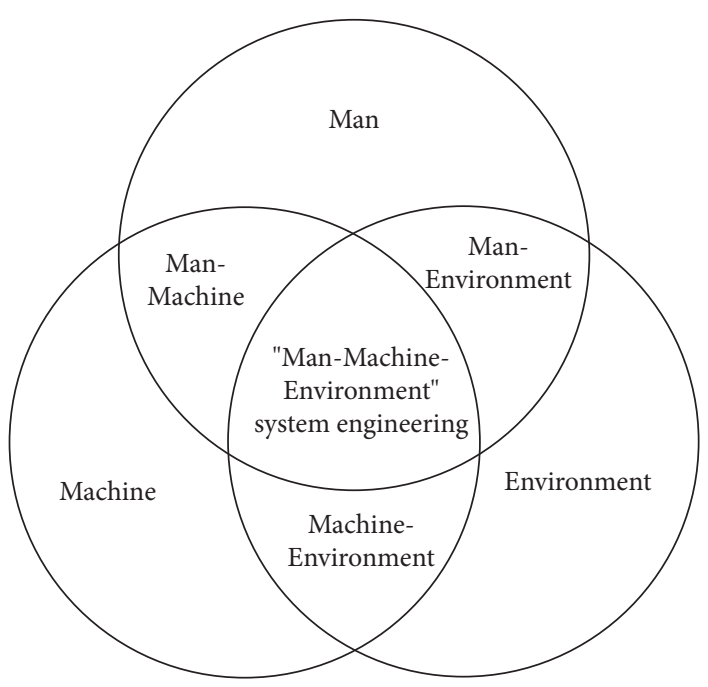

FIgure 1: Schematic diagram of the MMESE theory.

TABLE 1: MMESE talent growth factor index system (Human layer).

\begin{tabular}{|c|c|c|c|c|}
\hline Target level & $\begin{array}{l}\text { Criterion } \\
\text { level }\end{array}$ & Subtarget level & Attribute & Index level \\
\hline \multirow{4}{*}{$\begin{array}{l}\text { MMESE talent evaluation } \\
\text { index system }\end{array}$} & \multirow{4}{*}{$\begin{array}{l}\text { Human } \\
\quad(B 1)\end{array}$} & Health level $(C 1)$ & Qualitative & - \\
\hline & & $\begin{array}{l}\text { Psychological } \\
\text { quality }(C 2)\end{array}$ & Quantitative & Self-harmony, interpersonal harmony, and social harmony \\
\hline & & Education level (C3) & Qualitative & $\begin{array}{c}\text { The highest level of education currently received by the } \\
\text { individual }\end{array}$ \\
\hline & & $\begin{array}{l}\text { Innovation ability } \\
(C 4)\end{array}$ & Quantitative & $\begin{array}{l}\text { The ability to continuously provide new ideas, new } \\
\text { theories, new methods, and new inventions }\end{array}$ \\
\hline
\end{tabular}

The $C 5$ aspect reflects the amount of information acquired. According to the Statistical Report on China's Internet Development Status released by China Internet Network Information Center (CNNIC), as of December 2020, China holds 980 million Internet users $(70.4 \%$ of its total population). Moreover, there are 342 million online education users and 460 million online literature users, accounting for $34.6 \%$ and $46.5 \%$ of all the netizens, respectively. The average weekly online time per capita is 26.2 hours; meanwhile, the average monthly mobile phone traffic for an individual in China is $7.2 \mathrm{G}$, which is 1.2 times the global average. In summary, Internet has become an important source for people to receive knowledge and information.

The $C 6$ aspect focuses on how well an individual masters computer skills. In most industries, computers have become the primary tool to improve the efficiencies in industrial production, operation, and management and to reduce the operational risks and costs. Parameters in the C6 aspect reflect a person's capability of working with computers, for example, to what extent an individual masters common office software and network applications.

With the analysis above, the detailed indicators of the "Machine" layer in the MMESE talent evaluation index system are obtained (Table 2).
2.2.3. Factors of the B3 Layer. In the B3 layer "Environment," talents are evaluated from four aspects, which consist of the economic environment (C7), the policy environment $(C 8)$, the network environment (C9), and the family environment $(C 10)$.

The $C 7$ aspect addresses the economic factors. The economy casts varied effects on talent development. Specifically, the changes in the local consumption structure and residents' income could affect local investment in talent training. In this paper, GDP indicators are chosen to represent the impact of the economic environment on talents.

The $C 8$ aspect is devoted to the relevant policies. Regional policies can either restrict or promote talent development, and talent growth and development are inseparable from the principles of national and regional policies. Favorable policies can support talent growth by inspiring work enthusiasm in the local population or creating favorable social conditions for talent growth. In this paper, policies and laws about talent introduction, talent incentives, and talent protection are selected to describe the policy environment.

The $C 9$ aspect focuses on the computer network, which directly affects the way people obtain information. People in different regions have significantly different experiences in enjoying the convenience, diversity, and timeliness of 
TABle 2: MMESE talent growth factor index system (Machine layer).

\begin{tabular}{|c|c|c|c|c|}
\hline Target level & $\begin{array}{c}\text { Criterion } \\
\text { level }\end{array}$ & Subtarget level & Attribute & Index level \\
\hline \multirow{2}{*}{$\begin{array}{l}\text { MMESE talent } \\
\text { evaluation index system }\end{array}$} & \multirow{2}{*}{$\begin{array}{l}\text { Machine } \\
\text { (B2) }\end{array}$} & $\begin{array}{l}\text { Daily information } \\
\text { downloads }(C 5)\end{array}$ & & $\begin{array}{c}\text { The amount of information downloaded by individuals } \\
\text { on the Internet every day }\end{array}$ \\
\hline & & $\begin{array}{l}\text { Computer application } \\
\text { capabilities (C6) }\end{array}$ & Quantitative & $\begin{array}{l}\text { Including the use of common office software, network } \\
\text { applications, and handling simple problems }\end{array}$ \\
\hline
\end{tabular}

knowledge acquisition via the Internet. The network development level, a factor neglected in traditional talent growth index frameworks, is dynamic, innovative, and forward-looking. In this paper, local data download speed is chosen to define the network quality.

The $C 10$ aspect investigates the family factors. Families are the birthplaces and cradles of talents, and they are the space for individual growth and life, bringing fundamental impacts to the development of individual talents. Specifically, the literacy of family members is an important factor that affects individual development, which is therefore chosen as the parameter for C10.

With the analysis above, the detailed indicators of the "Machine" layer in the MMESE talent evaluation index system are obtained (Table 3 ).

In a word, the MMESE talent evaluation index system involves the impacts from the development of human intelligence, the machines, and the external environment on talents. Both humans and computers have their strengths and weaknesses. Computers are more effective in massive, repetitive, and programmed work, while humans are more productive in tasks that require advanced and creative thinking. When selecting new talent indicators to improve a talent evaluation system, the three major layers of MMESE should be studied in depth.

\section{Relevant Theories of Qualitative Index Weight Calculation Based on Probabilistic Language}

The comprehensive evaluation of a talent growth factor index system is a highly complex and uncertain decisionmaking problem, and its quantitative calculation is extremely difficult, making a reasonable and simplified model necessary to solve such a problem. Compared with traditional evaluation methods, hesitant fuzzy linguistic decisionmaking methods are very applicable when processing complex problems. Extensive studies have been performed on (group) decision-making based on hesitant fuzzy linguistic term sets. In this paper, the probabilistic language entropy theory is improved for the qualitative talent evaluation, and the weight calculation strategy for the qualitative criteria in probabilistic languages based on the entropy theory is proposed.

3.1. Probabilistic Language Term Set. The probabilistic language term set is expanded based on the hesitant fuzzy language term set. Decision-makers can provide multiple linguistic terms to state their opinions and their corresponding probability information to represent their comprehensive and accurate preferences.

Definition 1 (see [18]). Assume that $S=\left\{s_{t} \mid t=\right.$ $-\tau, \ldots,-1,0,1, \ldots \tau\}$ is a limited and completely ordered discrete language term set. As a result, it is a probabilistic language term set that can be expressed as

$$
h_{s}=\left\{s_{\phi_{l}}\left(p_{l}\right) \mid \quad s_{\phi_{l}} \in S, p_{l} \geq 0, l=1,2, \ldots, \# h_{s}, \sum_{l=1}^{\# h_{s}} p_{l} \leq 1\right\},
$$

where $s_{\phi_{1}}$ represents the language term in the language term set $S, p_{l}$ represents the probability of $s_{\phi_{l}}$, and $\# h_{s}$ is the number of language terms in $h_{s}$.

If $\sum_{l=1}^{\# h_{s}} p_{l}=1$, then we say that $h_{s}$ has complete probability distribution information; if $\sum_{l=1}^{\# h_{s}} p_{l}<1$, then some language term information in $h_{s}$ is unknown. In actual decision-making processes, because the experience and knowledge of experts are limited, incomplete information in $h_{s}$ is common. Besides, the probability information in $h_{s}$ could even be completely unknown if all the experts could not give the corresponding language terms.

If the language term information in $h_{s}$ is incomplete, then it would need normalization. Specifically, if a language term $s_{l}$ is not present in $h_{s}$, it should not appear in the probability language term set after normalization, either. Based on this idea, unknown probability information 1 $\sum_{l=1}^{\# h_{s}} p_{l}$ is evenly distributed to each language term in $h_{s}$.

Definition 2 (see [28]). $h_{s}=\left\{s_{\phi_{l}}\left(p_{l}\right) \mid s_{\phi_{l}} \in S, p_{l} \geq 0, l=1,2\right.$, $\left.\ldots, \# h_{s}, \sum_{l=1}^{\# h_{s}} p_{l} \leq 1\right\}$ is a set of probability language terms on a language term set $S$, and if $\sum_{l=1}^{\# h_{s}} p_{l}<1$, the normalized set of probability language terms $\widetilde{h}_{s}$ becomes

$$
\tilde{h}_{s}=\left\{s_{\phi_{l}}\left(p_{l}\right) \mid \quad s_{\phi_{l}} \in S, p_{l} \geq 0, l=1,2, \ldots, \# h_{s}, \sum_{l=1}^{\# h_{s}} p_{l}=1\right\} \text {, }
$$

where $\tilde{p}_{l}=\left(p_{l} / \sum_{l=1}^{\# h_{s}} p_{l}\right), l=1,2, \ldots, \# h_{s}$.

3.2. Comparison of the Probabilistic Language Term Sets. Symbolic distance, as a measure of information in fuzzy theory and fuzzy decision-making, is used to study the rankings of fuzzy numbers. Since fuzzy language term sets are simple and convenient to compare based on symbolic operations, this paper proposes a comparison strategy for 
probabilistic language term sets based on symbolic operations.

Definition 3 (see [28]). Assume $h_{s}=\left\{s_{\phi_{1}}\left(p_{l}\right) \mid s_{\phi_{l}} \in S, p_{l}\right.$ $\left.\geq 0, l=1,2, \ldots, \# h_{s}, \sum_{l=1}^{\# h_{s}} p_{l} \leq 1\right\}$ is a probabilistic language term set on a language term set $S=\left\{s_{t} \mid t=-\tau\right.$, $\ldots,-1,0,1, \ldots \tau\}, \# h_{s}$ is the number of language terms in $h_{s}$, and the function $I\left(s_{t}\right)$ can transform language terms $s_{t}$ into corresponding subscript values $t(t=-\tau, \ldots,-1,0,1, \ldots \tau)$. Then, the expectation of $h_{s}$ can be defined as

$$
e\left(h_{s}\right)=\sum_{l=1}^{\# h_{s}} I\left(s_{\phi_{l}}\right) p_{l} .
$$

Furthermore, the variance of $h_{s}$ is defined as

$$
v\left(h_{s}\right)=\sum_{l=1}^{\# h_{s}}\left(I\left(s_{\phi_{l}}\right)-e\left(h_{s}\right)\right)^{2} p_{l} .
$$

For any two probabilistic language term sets $h_{s}^{1}$ and $h_{s}^{2}$,

$$
\begin{aligned}
& h_{s}^{1}=\left\{s_{\phi_{l}}^{1}\left(p_{l}^{1}\right) \mid \quad s_{\phi_{l}}^{1} \in S, p_{l}^{1} \geq 0, l=1,2, \ldots, \# h_{s}^{1}, \sum_{l=1}^{\# h_{s}^{1}} p_{l}^{1} \leq 1\right\} ; \\
& h_{s}^{2}=\left\{s_{\phi_{l}}^{2}\left(p_{l}^{2}\right) \mid \quad s_{\phi_{l}}^{2} \in S, p_{l}^{2} \geq 0, l=1,2, \ldots, \# h_{s}^{2}, \sum_{l=1}^{\# h_{s}^{2}} p_{l}^{2} \leq 1\right\} \text {, }
\end{aligned}
$$

they can be compared based on their expectations and variances:

(1) If $e\left(h_{s}^{1}\right)>e\left(h_{s}^{2}\right)$, then $h_{s}^{1}$ is greater than $h_{s}^{2}$, which is recorded as $h_{s}^{1}>h_{s}^{2}$.

(2) If $e\left(h_{s}^{1}\right)<e\left(h_{s}^{2}\right)$, then $h_{s}^{1}$ is smaller than $h_{s}^{2}$, which is recorded as $h_{s}^{1} \prec h_{s}^{2}$.

(3) If $e\left(h_{s}^{1}\right)=e\left(h_{s}^{2}\right)$, the variances $v\left(h_{s}^{1}\right)$ and $v\left(h_{s}^{2}\right)$ should be calculated. If $v\left(h_{s}^{1}\right)>v\left(h_{s}^{2}\right)$, then $h_{s}^{1} \prec h_{s}^{2}$; if $v\left(h_{s}^{1}\right)<v\left(h_{s}^{2}\right)$, then $h_{s}^{1}>h_{s}^{2}$; if $v\left(h_{s}^{1}\right)=v\left(h_{s}^{2}\right), h_{s}^{1}$ is equivalent to $h_{s}^{2}$, which is recorded as $h_{s}^{1} \sim h_{s}^{2}$.

3.3. Probability Language Entropy. Probability language term sets are an extension of hesitant fuzzy language term sets, so probability language entropy, which is defined below, is also an extension of hesitant fuzzy language entropy and the probability language terminologies.

Definition 5 (see [29]). $S=\left\{s_{t} \mid t=-\tau, \ldots,-1,0,1, \ldots \tau\right\}$ is a set of language terms, $h_{s}=\left\{s_{\phi_{l}}\left(p_{l}\right) \mid s_{\phi_{l}} \in S, l=1,2, \ldots, \# h_{s}\right\}$, $h_{s}^{1}=\left\{s_{\phi_{l}}^{1}\left(p_{l}^{1}\right) \mid s_{\phi_{l}}^{1} \in S, l=1,2, \ldots, \# h_{s}^{1}\right\}, \quad$ and $h_{s}^{2}=\left\{s_{\phi_{l}}^{2}\left(p_{l}^{2}\right)\right.$ $\left.\mid s_{\phi_{l}}^{2} \in S, l=1,2, \ldots, \# h_{s}^{2}\right\}$ are three sets of probability language terms on $S, \# h_{s}=\# h_{s}^{1}=\# h_{s}^{2}=L$, and function $I\left(s_{t}\right)$ can convert language terms $S_{t}$ into the corresponding subscript values $t(t=-\tau, \ldots,-1,0,1, \ldots \tau)$. Then, a probability language entropy $E\left(h_{s}\right)$ must satisfy the following conditions:

(1) $0 \leq E\left(h_{s}\right) \leq 1$

(2) When and only when $h_{s}=\left\{S_{-\tau}(1)\right\}$ or $h_{s}=\left\{S_{\tau}(1)\right\}$, $E\left(h_{s}\right)=0$

(3) When and only when $\# h_{s}=2, p_{\phi_{1}}=p_{\phi_{2}}=0.5, I\left(s_{\phi_{1}}\right)+I\left(s_{\phi_{2}}\right)$ $=0, E\left(h_{s}\right)=1$

(4) For $\forall l=1,2, \ldots, L$, if or $I\left(s_{\phi_{1}}^{1}\right) \leq I\left(s_{\phi_{1}}^{2}\right) \leq 0$, and $p_{l}^{1}=p_{l}^{2}$, then $E\left(h_{s}^{1}\right) \geq E\left(h_{s}^{2}\right)$

(5) $E\left(h_{s}\right)=E\left(h_{s}^{c}\right)$, $h_{s}^{c}=\left\{s_{-\phi_{l}}\left(p_{l}\right)|| s_{\phi_{l}} \in S, p_{l} \geq 0, l=1,2, \ldots, \# h_{s}\right\}$

while

Based on these conditions, for any $h_{s}=\left\{s_{\phi_{l}}\left(p_{l}\right) \mid s_{\phi_{l}} \in S, p_{l} \geq 0, l=1,2, \ldots, \# h_{s}, \sum_{l=1}^{\# h_{s}} p_{l} \leq 1\right\}$ on $S=\left\{s_{t} \mid t=-\tau, \ldots,-1,0,1, \ldots \tau\right\}, \# h_{s}=L, E\left(h_{s}\right)$ of $h_{s}$ can be expressed as

$$
\begin{aligned}
E\left(h_{s}\right)= & -\frac{1}{2 L \ln 2} \sum_{l=1}^{L}\left[p_{l} \ln p_{l}+\left(1-p_{l}\right) \ln \left(1-p_{l}\right)+\frac{I\left(s_{\phi_{l}}\right)+I\left(s_{\phi_{(L-l+1)}}\right)+2 \tau}{4 \tau}\right. \\
& \left.\quad \ln \frac{I\left(s_{\phi_{l}}\right)+I\left(s_{\phi_{(L-l+1)}}\right)+2 \tau}{4 \tau}+\frac{2 \tau-I\left(s_{\phi_{l}}\right)-I\left(s_{\phi_{(L-l+1)}}\right)}{4 \tau} \ln \frac{2 \tau-I\left(s_{\phi_{l}}\right)-I\left(s_{\phi_{(L-l+1)}}\right)}{4 \tau}\right] .
\end{aligned}
$$

\section{The Comprehensive, Integrated, Qualitative, and Quantitative Decision-Making Model}

4.1. Problem Description. To solve multiattribute complex decision-making problems, experts need to consider the qualitative and quantitative properties of the objects and sort out the pros and cons of all the possible alternatives.
Specifically, quantitative attributes are generally expressed as specific values, while qualitative attributes are evaluated by the experts' language evaluations based on their empirical knowledge. In actual decision-making process, such language evaluations are summarized into a set of probability language terms, and because expert knowledge is limited, attribute weights are often inaccurate or even completely 
TABLE 3: MMESE talent growth factor index system (Environment layer).

\begin{tabular}{|c|c|c|c|c|}
\hline Target level & Criterion level & Subtarget level & Attribute & Index level \\
\hline \multirow{4}{*}{$\begin{array}{l}\text { MMESE talent evaluation index } \\
\text { system }\end{array}$} & \multirow{4}{*}{$\begin{array}{l}\text { Environment } \\
\text { (B3) }\end{array}$} & $\begin{array}{l}\text { Economic environment } \\
\text { (C7) }\end{array}$ & Qualitative & - \\
\hline & & Policy environment $(C 8)$ & Quantitative & - \\
\hline & & $\begin{array}{c}\text { Network environment } \\
\text { (C9) }\end{array}$ & Qualitative & Regional network speed \\
\hline & & Family environment $(C 4)$ & Qualitative & $\begin{array}{l}\text { Educational status of immediate family } \\
\text { members }\end{array}$ \\
\hline
\end{tabular}

unknown. Therefore, a multicriteria decision-making problem involving both qualitative and quantitative attributes can be described as follows.

Assume an alternative set $A=\left\{A_{1}, A_{2}, \ldots, A_{m}\right\}$. Its decision criteria contain quantitative $C^{z}=\left\{C_{1}^{z}, C_{2}^{z}, \ldots, C_{n_{z}}^{z}\right\}$ and qualitative $C^{q}=\left\{C_{1}^{q}, C_{2}^{q}, \ldots, C_{n_{q}}^{q}\right\}$ property sets, and the corresponding criterion attribute weight sets are $w^{z}=\left(w_{1}^{z}, w_{1}^{z}, \ldots, w_{n_{z}}^{z}\right)$ and $w^{q}=\left(w_{1}^{q}, w_{1}^{q}, \ldots, w_{n_{q}}^{q}\right)$, respectively. The criterion attribute weight sets must satisfy $\sum_{j=1}^{n_{z}} w_{j}^{z}+\sum_{k=1}^{n_{q}} w_{k}^{q}=1$, and all guidelines are considered preferred. Then, consider a property value matrix $X=\left(x_{i j}\right)_{m \times n_{z}}\left(i=1,2, \ldots, m ; j=1,2, \ldots, n_{z}\right)$ under $C^{z}$, in which $x_{i j}$ represents the property value of $A_{i}$ on the quantitative attribute $C_{j}^{z}$. Subsequently, a probability language decision matrix $H=\left(h_{s_{i k}}\right)_{m \times n_{q}}\left(i=1,2, \ldots, m ; k=1,2, \ldots, n_{q}\right)$ could be obtained by summarizing the expert decision information under $C^{q}$, in which $h_{s_{i k}}$ represents the probability language term set of $A_{i}$ on $C_{k}^{q}$.

In this paper, the weights of all criteria attributes are assigned as completely unknown to avoid inaccurate weights in the decision-making process. Consequently, a qualitative attribute weight set $w^{z}=\left(w_{1}^{z}, w_{1}^{z}, \ldots, w_{n_{z}}^{z}\right)$ and a quantitative attributes weight set $w^{q}=\left(w_{1}^{q}, w_{1}^{q}, \ldots, w_{n_{q}}^{q}\right)$ must be obtained via reasonable calculations. In addition, all criteria attributes must be integrated to obtain the final decisions for complex decision-making problems. This paper proposes a comprehensive and integrated decision-making method based on probabilistic hesitation language entropy, crossentropy, and the possibility of priority relationship, and this method could provide qualitative and quantitative solutions to complex decision-making problems in talent evaluation.

\subsection{Property Weight Calculations Based on Entropy. The} entropy weight method obtains the entropy information and calculates the attribute weights simply based on the data of each decision attribute, thereby evading the subjectivity of attribute weight assignments. In this paper, the entropy weight method is applied to calculate the quantitative attribute weights following a previous report [30], and the calculated results would be incorporated with the probabilistic language entropy theory to obtain the qualitative attribute weights. The specific calculation process of quantitative attribute weights has been elaborated in literature [31], which will not be repeated here.

For the weight calculation of qualitative attributes, the probability language entropy $E\left(h_{s_{k}}\right)$ is calculated following formula (5), and the total entropy $E_{k}=\sum_{i=1}^{m} E\left(h_{s_{i k}}\right)$ of all the qualitative attributes $C_{k}^{q}$ is obtained by accumulation. According to the entropy theory, smaller overall entropy numbers correspond to larger attribute weights. Therefore, we have the calculation formula for qualitative attribute weights:

$$
w_{k}^{q}=\frac{\left(\sum_{k=1}^{k=n_{q}} \widehat{E}_{k}\right)}{\sum_{k=1}^{k=n_{q}}\left(\left(\sum_{k=1}^{k=n_{q}} \widehat{E}_{k}\right) / \widehat{E}_{k}\right)} .
$$

\subsection{Alternative Sorting Based on the Possibility of Precedence} Relationship. In a multicriteria decision-making problem, the criterion attribute values of each alternative should be integrated for comprehensive ranking. However, the relationship between the pros and cons of the alternatives can be determined by comparing their attribute values in single criteria. Specifically, Gou et al. [30] proposed an alternative queuing method (AQM) based on the outranking method, which processes the priority relationships with a directed relationship graph and a 0 -1 matrix. In this paper, a ranking method for alternatives is proposed based on the possibility of priority relationships [31,32].

First, the directed relationship graph and the $0-1$ matrix of the optimal order relationship among the alternatives must be explained. For two alternatives $A_{i}$ and $A_{j}$, if $A_{i}>A_{j}$ ( $A_{i}$ better than $A_{j}$ ), their directed relationship can be expressed as $A_{i} \longrightarrow A_{j}$ in the graph; if $A_{i}=A_{j}$ (the priorities of $A_{i}$ and $A_{j}$ are equal), their directed relationship can be expressed as $A_{i} \cup A_{j}$ in the graph. Finally, if the advantages and disadvantages of $A_{i}$ and $A_{j}$ are not comparable, their relationship shall not be represented in the directed relationship graph. Similarly, a 0-1 matrix can be applied to describe the relationships. Assume that the $0-1$ matrix is $M$. If $A_{i}>A_{j}$, make $M_{i j}=1, M_{i j}=0$; if $A_{i}=A_{j}$, make $M_{i j}=M_{j i}=1$; if the pros and cons of $A_{i}$ and $A_{j}$ are not comparable, then make $M_{i j}=M_{j i}=0$. In particular, the priority relationship of the alternatives in the $0-1$ matrix is expressed as 1; in other words, the elements on the main diagonal always satisfy $M_{i j}=M_{j i}=1$ in the $0-1$ matrix.

The priority relationship between four sample alternatives $A_{1}, A_{2}, A_{3}, A_{4}$ under a single criterion can be represented by the directed graph (Figure 2 ).

The order of priority relationships in Figure 2 is used for illustration. The probability of association among $A_{i}$ and $A_{j}$ is matrix $P$ which is used to indicate the credibility of the situation that $A_{i}$ is associated with $A_{j}$. The four alternatives 


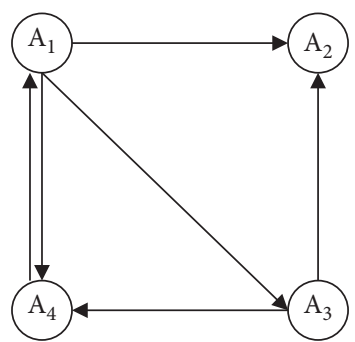

FIgURE 2: The directed graph of alternative plans.

$A_{1}, A_{2}, A_{3}, A_{4}$ are compared in pairs to get four possibility matrices $(P s)$ that describe their mutual priority relationships:

$$
\begin{aligned}
& A_{1} \\
& P=A_{2} \\
& A_{2} \\
& A_{3} \\
& A_{4}
\end{aligned} \quad\left(\begin{array}{llll}
p_{11} & p_{12} & p_{13} & p_{14} \\
p_{21} & p_{22} & p_{23} & p_{24} \\
p_{31} & p_{32} & p_{33} & p_{34} \\
p_{41} & p_{42} & p_{43} & p_{44}
\end{array}\right) .
$$

An element $p_{i j}(i, j=1,2,3,4)$ in a possibility degree matrix $P$ must satisfy the following properties:

(1) $0 \leq p_{i j} \leq 1$

(2) $p_{i j}+p_{j i}=1$, especially, $p_{i i}=0.5(i, j=1,2,3,4)$

In a possibility degree matrix $P=\left(P_{i j}\right)_{m \times m}(i, j=1,2, \ldots, m)$ that reflects the priority relationships among $m$ alternatives, the possible degree information of all $m$ alternatives is mutually compared, and $P$ is a fuzzy complementary judgment matrix. Then, the problem of ranking the pros and cons of the $m$ alternatives turns into a problem of finding the ranking vector of $P$, which has been solved in a previous report [31]:

$$
v_{i}=\frac{1}{m(m-1)}\left(\sum_{j=1}^{m} p_{i j}+\frac{m}{2}-1\right), \quad(i=1,2, \ldots, m) .
$$

The ranking vector $V=\left(v_{1}, v_{2}, \ldots, v_{m}\right)^{T}$ of the possibility degree matrix $P=\left(P_{i j}\right)_{m \times m}$ can be obtained by formula (10), and the pros and cons of the $m$ alternatives can be ranked by the numerical values of the elements in $V$.

4.4. Comprehensive, Integrated, Qualitative, and Quantitative Decision-Making. To solve multicriteria decision-making problems with both qualitative and quantitative attributes, entropy weight, probabilistic language entropy, cross-entropy, and priority ordering methods are integrated to propose a comprehensive 3-part decision-making method.

(1) Calculate the quantitative attribute weight set $w^{z}$ and the qualitative attribute weight set $w^{q}$.

(2) Choose an appropriate weight integration coefficient $\delta(\delta>0)$ so that $\left(\sum w^{z} / \sum w^{q}\right)=\delta$. Then the standard weight set becomes

$$
w=\left(w_{1}, w_{2}, \ldots, w_{n_{z}}, w_{n_{z}+1}, w_{n_{z}+2}, \ldots, w_{n_{z}+n_{q}}\right),
$$

where $\quad w_{i}=\left(w^{z}(i) /(1+\delta)\right),\left(i=1,2, \ldots, n_{z}\right), \quad w_{n_{z}+j}=$ $\left(\delta w^{q}(j) /(1+\delta)\right),\left(j=1,2, \ldots, n_{q}\right)$, and $n_{z}$ and $n_{q}$ are the quantitative attributes and their quantities, respectively.

Since the weights of qualitative and quantitative attributes are calculated via different methods, they are not directly comparable. Besides, $\delta(\delta>0)$ is a factor that directly affects the conversion of the standard weight set $w=\left(\bar{w}^{z}, \bar{w}^{q}\right)$. Meanwhile, since the importance of each attribute in actual decision-making processes cannot be too wide, the differences in importance among attributes will generally not exceed 10 times. Therefore, if the decisionmaker does not have a clear preference on the importance of attributes, $\delta$ values may be selected within $0.1 \leq \delta \leq 10$ (when $0.1 \leq \delta<1$, quantitative attributes are more stressed; when $1<\delta \leq 10$, then qualitative attributes are more stressed). Following that, calculate the change interval $\left[R_{i}^{-}, R_{i}^{+}\right]$of the overall pros and cons $R_{i}$ of scheme $A_{i}$, where $R_{i}^{-}$and $R_{i}^{+}$ represent the minimum and maximum values of $R_{i}$ across the alternatives, respectively. Compare the change intervals of $R_{i}$ of all alternatives $A_{i}(i, j=1,2, \ldots, m)$ in pairs to obtain the possibility degree matrix $P=\left(P_{i j}\right)_{m \times m}(i, j=1,2, \ldots, m)$ that describes the priority relationships across all alternatives and obtain the comprehensive rankings of the alternatives with the ranking method in Section 4.3.

The overall steps of the proposed method are as follows:

Step 1: calculate the quantitative attribute weight set $w^{z}$ and the qualitative attribute weight set $w^{q}$ according to Section 4.2, and $\sum w^{z}=1, \sum w^{q}=1$.

Step 2: set the value interval of $\delta$ as $\delta \in[0.1,10]$, and take $\delta=0.1$ as the initial weight integration coefficient.

Step 3: input $\delta$, and calculate the standard weight set $w=\left(\bar{w}^{z}, \bar{w}^{q}\right)$.

Step 4: under each criterion attribute $C_{j}$ (when $j=1,2, \ldots, n_{z}$, it refers to the quantitative attributes; when $j=n_{z}+1, n_{z}+2, \ldots, n_{z}+n_{q}$, it refers to the qualitative attributes), compare the alternatives in pairs and establish a directed graph or 0-1 matrix for them. For quantitative attributes, the values of the attributes may be compared directly to determine the pros and cons of the alternatives. For qualitative attributes, the pros and cons of the alternatives may be compared following the probabilistic language term set method in Section 2.2. The 0-1 matrices are constructed following the procedure and definitions in Section 4.3.

Step 5: based on the prioritized matrix $M_{j}$ obtained in step 4 , for alternatives $A_{i}, A_{k}(i, k=1,2, \ldots, m)$, the cumulative weight of all the criterion attributes that satisfy $A_{i}>A_{k}$ is recorded as the overall benefit weight $w_{\succ}(i, k)$; similarly, the cumulative weight of all the criterion attributes that satisfy $A_{i} \prec A_{k}$ is recorded as the overall disadvantage weight $w_{\prec}(i, k)$, and the 
cumulative weight of all the criterion attributes that satisfy $A_{i}=A_{k}$ is recorded as the overall indifference weight $w_{=}(i, k)$.

Step 6: construct alternative plans for the indicators for the measurement of $A_{i}, A_{k}(i, k=1,2, \ldots, m)$ 's pros and cons degree:

$$
R_{i k}=\frac{w_{\succ}(i, k)+\mu w_{\approx}(i, k)}{w_{\succ}(i, k)+(1-\mu) w_{\approx}(i, k)},
$$

where $0 \leq \mu \leq 1$ indicates the influence degree of the alternatives and the indifference situation on the pros and cons. Larger $R_{i k}$ suggests higher superiority of $A_{i}$ over $A_{k}$.

Step 7: calculate the average $R_{i k}$ of the alternatives $A_{i}, A_{k}$ and the overall pros and cons $R_{i}$ of the alternative $A_{i}$ :

$$
R_{i}=\frac{1}{m} \sum_{k=1}^{m} R_{i k}
$$

Step 8: update the maximum and minimum values in $\left[R_{i}^{-}, R_{i}^{+}\right]$for all alternatives $A_{i}$, and let $\delta=\delta+0.01$. If $\delta>10$, go to step 9; otherwise, go to step 3 .

Step 9: compare the interval $\left[R_{i}^{-}, R_{i}^{+}\right]$of each pair according to formula (13), and obtain the probability $p_{i j}(i, j=1,2, \ldots, m)$ of plan $A_{i}$ being superior over $A_{j}$ $[33,34]$ :

$$
p_{i j}=\frac{\min \left\{L_{i}+L_{j}, \max \left(R_{i}^{+}-R_{j}^{-}, 0\right\}\right.}{L_{i}+L_{j}},
$$

where $L_{i}=R_{i}^{+}-R_{j}^{-}, L_{j}=R_{j}^{+}-R_{j}^{-}$.

Step 10: build the possible degree matrix $P=\left(p_{i j}\right)_{m \times n}$ based on the $p_{i j}(i, j=1,2, \ldots, m)$ numbers obtained for all alternatives, and rank the alternatives with the ranking method in Section 4.3.

\section{Case Analysis of the Proposed Method}

5.1. Case Description. In this study, the qualitative and quantitative parameters of three persons $A_{1}, A_{2}$, and $A_{3}$ were comprehensively evaluated with the proposed method, and their pros and cons were ranked. The language term set $S=$ $\left\{s_{-3}=\right.$ extreme poor, $s_{-2}=$ verybad, $s_{-1}=$ poor, $s_{0}$ $=$ fair, $s_{1}=$ good, $s_{2}=$ very good, $s_{3}=$ excellent $\}$ was used to evaluate the persons.

$C_{1}$ results in Table 4 were obtained based on personal health risk assessments following common medical procedures, and the overall personal health risk $W$ was calculated by dividing the health risk values. Under normal circumstances, $W$ is divided into seven levels, described in a set $W=\left\{W_{-3}=\right.$ no risk, $W_{-2}=$ very low risk, $W \quad-1=$ low risk, $W_{0}=$ general risk, $W_{1}=$ medium risk, $W_{2}=$ high risk, $W_{3}=$ major risk $\} . C_{3}$ and $C_{10}$ results are based on the highest education levels of the individuals and their immediate family members. According to Regulations of the People's Republic of
China on Academic Degrees, the results can be described by any element in the following set: $E=\left\{E_{3}=\right.$ doctorate, $E_{2}=$ master' s degree, $E_{1}=$ undergraduate, $E_{0}=$ university, $E_{-1}=$ senior high school, $E_{-2}=$ junior high school, $E_{-3}=$ primaryschool\}. $C_{5}$ results were obtained based on the average daily network usage. $C_{7}$ data reflect the local per capita GDP at the individual's residence, and $C_{9}$ data were obtained based on the individual's local area network. With these data, the quantitative index data of the three talents evaluated were obtained (Table 4).

Table 5 shows the qualitative judgment information of 5 experts in terms of the indicators in $C 2, C 4, C 6$, and $C 8$ aspects on the 3 talents evaluated.

The information in Table 5 was processed to obtain the language terms and their corresponding probability values (Table 6 and 7).

The analysis of the cases above followed the methods discussed in this paper, and the specific process is described as follows:

Step 1: calculate the weight sets of quantitative indicators $C_{1}, C_{3}, C_{5}, C_{7}, C_{9}, C_{10}$ by the entropy weights $w_{z}=(0.1502,0.1598,0.1717,0.2157,0.1428)$ according to formulas (8) and (9). Similarly, calculate the weight sets of qualitative indicators $C_{2}, C_{4}, C_{6}, C_{8}$ by the entropy weights $w_{q}=(0.2609,0.2443,0.2939$, 0.2008).

Steps 2 to 8 : set the parameter $\mu=0.6$ and the value interval of the weight integration coefficient $\delta=[0.1,10]$. Subsequently, update the values of $\delta$ with a step width of 0.01 , and calculate the changes in the pros and cons of the qualitative and quantitative evaluation of talents $\left[R_{i}(i=1,2,3)\right]$ as described in Figure 3.

In order to visually display the distribution of $R_{i}(i=$ $1,2,3)$ as $\delta$ changes, a box plot is depicted (Figure 4).

$\left[R_{i}^{-}, R_{i}^{+}\right]$of the qualitative and quantitative evaluation and talent rankings are obtained as follows for the three evaluated personnel:

$$
\begin{aligned}
& {\left[R^{-}, R^{+}\right]=[1.5809,1.7874] ;} \\
& {\left[R^{-}, R^{+}\right]=[2.4639,3.4846] ;} \\
& {\left[R^{-}, R^{+}\right]=[0.6575,0.7268] .}
\end{aligned}
$$

Steps 9 to 10: compare the overall $R_{i}(i=1,2,3)$ for all the talents in pairs, and obtain the probability matrix $P$ that describes the priority relationship of talent ranking with formula (13):

$$
P=\left(\begin{array}{ccc}
0.5 & 0 & 1 \\
1 & 0.5 & 1 \\
0 & 0 & 0.5
\end{array}\right)
$$

According to formula (10), the ranking vector $V$ of $P$ was calculated to be 
TABLE 4: Quantitative attribute value matrix for comprehensive talent evaluation.

\begin{tabular}{cccccc}
\hline & $C_{1}$ & $C_{3}$ & $C_{5}(\mathrm{~GB})$ & $C_{7}$ (ten thousand/yuan) & $C_{9}(\mathrm{Mbps})$ \\
\hline$A_{1}$ & $W_{2}$ & $E_{1}$ & 1.5 & 8.78 & 47.44 \\
$A_{2}$ & $W_{-2}$ & $E_{3}$ & 2.5 & 16.49 & 97.66 \\
$A_{3}$ & $W_{0}$ & $E_{0}$ & 1 & 6.62 & $E_{-1}$ \\
\hline
\end{tabular}

TABLE 5: Language decision matrix provided by decision experts.

\begin{tabular}{|c|c|c|c|c|c|}
\hline & & $C_{2}$ & $C_{4}$ & $C_{6}$ & $C_{8}$ \\
\hline \multirow{3}{*}{ Expert 1} & $A_{1}$ & $S_{2}$ & $S_{0}$ & $S_{1}$ & $S_{2}$ \\
\hline & $A_{2}$ & $S_{0}$ & $S_{3}$ & $S_{2}$ & $S_{2}$ \\
\hline & $A_{3}$ & $S_{3}$ & $S_{1}$ & $S_{-2}$ & $S_{0}$ \\
\hline \multirow{3}{*}{ Expert 2} & $A_{1}$ & $S_{1}$ & $S_{-1}$ & $S_{0}$ & $S_{2}$ \\
\hline & $A_{2}$ & $S_{-1}$ & $S_{2}$ & $S_{3}$ & $S_{3}$ \\
\hline & $A_{3}$ & $S_{2}$ & $S_{-2}$ & $S_{-3}$ & $S_{0}$ \\
\hline \multirow{3}{*}{ Expert 3} & $A_{1}$ & $S_{1}$ & $S_{1}$ & $S_{2}$ & $S_{1}$ \\
\hline & $A_{2}$ & $S_{-2}$ & $S_{2}$ & $S_{1}$ & $S_{2}$ \\
\hline & $A_{3}$ & $S_{2}$ & $S_{1}$ & $S_{-2}$ & $S_{-1}$ \\
\hline \multirow{3}{*}{ Expert 4} & $A_{1}$ & $S_{3}$ & $S_{-1}$ & $S_{2}$ & $S_{2}$ \\
\hline & $A_{2}$ & $S_{-1}$ & $S_{2}$ & $S_{2}$ & $S_{3}$ \\
\hline & $A_{3}$ & $S_{2}$ & $S_{1}$ & $S_{-1}$ & $S_{1}$ \\
\hline \multirow{3}{*}{ Expert 5} & $A_{1}$ & $S_{2}$ & $S_{2}$ & $S_{-1}$ & $S_{2}$ \\
\hline & $A_{2}$ & $S_{-2}^{2}$ & $S_{3}$ & $S_{3}$ & $S_{2}$ \\
\hline & $A_{3}$ & $S_{2}$ & $S_{0}$ & $S_{-1}$ & $S_{0}$ \\
\hline
\end{tabular}

TABLE 6: Linguistic terms and their corresponding probability values $\left(C_{2}, C_{4}\right)$ after summary of expert decision information.

\begin{tabular}{lcc}
\hline & $C_{2}$ & $C_{4}$ \\
\hline$A_{1}$ & $\left\{S_{1}(0.4), S_{2}(0.4), S_{3}(0.2)\right\}$ & $\left\{S_{-1}(0.4), S_{0}(0.2), S_{1}(0.2), S_{2}(0.2)\right\}$ \\
$A_{2}$ & $\left\{S_{-2}(0.4), S_{-1}(0.4), S_{0}(0.2)\right\}$ & $\left\{S_{2}(0.6), S_{3}(0.4)\right\}$ \\
$A_{3}$ & $\left\{S_{2}(0.8), S_{3}(0.2)\right\}$ & $\left\{S_{-2}(0.2), S_{0}(0.2), S_{1}(0.6)\right\}$ \\
\hline
\end{tabular}

TABLE 7: Comparison of results from different decision-making methods.

\begin{tabular}{lccc}
\hline Decision method & Decision index & Alternative ranking results & Optimal alternative \\
\hline$[35]$ & $\mathbf{C}_{1}, \mathbf{C}_{3}, \mathbf{C}_{5}, \mathbf{C}_{7}, \mathbf{C}_{9}, \mathbf{C}_{10}$ & $\mathbf{A}_{1}>\mathbf{A}_{2}>\mathbf{A}_{3}$ & $\mathbf{A}_{3}$ \\
{$[19]$} & $\mathbf{C}_{2}, \mathbf{C}_{4}, \mathbf{C}_{6}, \mathbf{C}_{8}$ & $\mathbf{A}_{2}>\mathbf{A}_{1}>\mathbf{A}_{3}$ & $\mathbf{A}_{2}$ \\
{$[20]$} & $\mathbf{C}_{1}, \mathbf{C}_{2}, \mathbf{C}_{3}, \mathbf{C}_{4}, \mathbf{C}_{5}, \mathbf{C}_{6}, \mathbf{C}_{7}, \mathbf{C}_{8}, \mathbf{C}_{9}, \mathbf{C}_{10}$ & $\mathbf{A}_{2}>\mathbf{A}_{1}>\mathbf{A}_{3}$ & $\mathbf{A}_{2}$ \\
Paper method & $\mathbf{C}_{1}, \mathbf{C}_{2}, \mathbf{C}_{3}, \mathbf{C}_{4}, \mathbf{C}_{5}, \mathbf{C}_{6}, \mathbf{C}_{7}, \mathbf{C}_{8}, \mathbf{C}_{9}, \mathbf{C}_{10}$ & $\mathbf{A}_{2}>\mathbf{A}_{1}>\mathbf{A}_{3}$ & $\mathbf{A}_{2}$ \\
\hline
\end{tabular}

$$
V=(0.3333,0.5000,0.1667)^{T} \text {. }
$$

Judging from the elements in $V$, the final qualitative and qualitative comprehensive evaluation result for the three talents was $A_{2}>A_{1}>A_{3}$. Additionally, with the possibility degree matrix $P$, a comprehensive ranking including the possibility degree of the optimal order relationship revealed that all probabilities of superiority were 1 , and the evaluation result remained $A_{2} \succ A_{1} \succ A_{3}$.

5.2. Method Comparison. In this section, the method proposed in this paper was compared with those that use either qualitative or quantitative indicators alone for talent evaluation with a comprehensive entropy-based evaluation strategy [33], and the quantitative indicators in Section 5.1 were used as the samples. More specifically, the maximum deviation-based decision method described in literature [19] was used to sort out the alternatives in the qualitative indicators of the 5.1 case, and a previously reported method [20] and the method proposed in this paper were used to sort the evaluation objects through all the quantitative and qualitative indicators. The comparative analysis of talent rankings is described in Table 7.

From Table 7, it could be concluded that the decisionmaking results from quantitative indicators alone are quite different from those from other methods. More precisely, the decision-making results from quantitative indicators are more sensitive to the influence of simple data and information. On the other hand, the comprehensive talent evaluation is a complex process, which asks for comprehensive consideration of the internal and external factors in talent growth, but the decision-making results from quantitative data alone are more one-sided, making them 


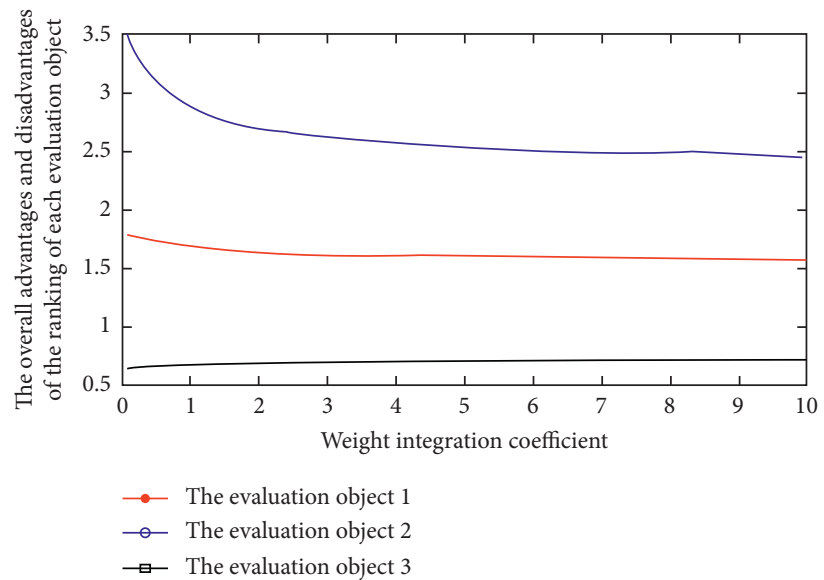

Figure 3: The changes of the weight integration coefficient $\delta$ and the overall pros and cons of each person's ranking.

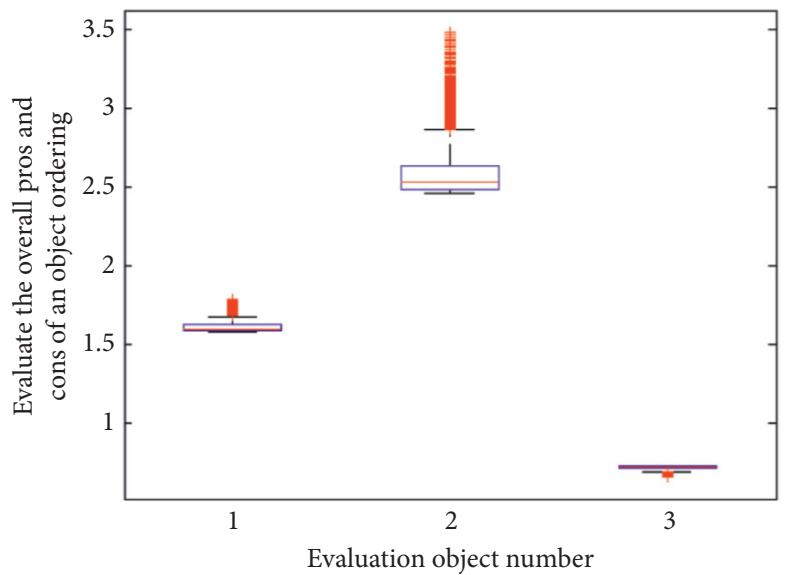

FIgURE 4: The distribution box plot of the overall pros and cons of talent ranking.

inaccurate. For decision-making using qualitative indicators alone, the produced results obtained via the maximum deviation method are identical to those obtained via averaging the qualitative and quantitative integrated weights, suggesting that experts can use their own experience and knowledge comprehensively during complex problem decision-making to produce more comprehensive judgments.

Even so, the decision-making method in this paper can not only get the rankings of alternatives without predefining qualitative and quantitative weight integration coefficients but also obtain the possibility of the priority relationship between two alternatives, which is an intuitive reflection of the relationship between the alternatives. Meanwhile, since the comprehensive talent evaluation method proposed in this paper considers the comprehensive influence of quantitative and qualitative attributes on the talent evaluation results, the evaluation process is more reasonable and applicable to heterogeneous index data.

\section{Conclusion}

A comprehensive qualitative and quantitative talent evaluation index system is proposed in this paper for talent evaluation in complex environments. The proposed system is based on the "human-machine-environment" system engineering theory, the probabilistic language entropy, and priority relationships. There are three main research conclusions:

(1) The coupling relationships between human and machine, human and environment, and environment and machine were analyzed to establish a talent evaluation index system that integrates MMESE qualitative and quantitative factors. The system not only reflects the use of machines in the information age to promote talent growth but also reflects the impact of the external environment on talents. The proposed system is more favorable than previously available talent evaluation index systems because it considers both qualitative and quantitative aspects instead of just one.

(2) Theories related to probabilistic language terminology were applied to qualitative indicators of talents as assessed by experts. These theories can reflect the vagueness and uncertainty of experts' knowledge-based empirical judgments more 
accurately so that more accurate expert evaluation results could be obtained.

(3) The entropy theory was used to calculate the attribute weights of qualitative criteria, and by integrating the theory with the possibility matrix of the priority relationships, a comprehensive integrated decision-making method that involves both qualitative and quantitative factors in talent evaluation is proposed to consider statistical data indicators and expert knowledge for more promising and accurate talent evaluation. The talent evaluation results obtained from the method are more consistent with the real-world situation. The validity of the decisionmaking method in this paper is verified with the examples and the comparative analysis of the available methods.

In a word, the MMESE talent evaluation index system and comprehensive evaluation method proposed in this paper can provide scientific and accurate solutions for talent evaluation and selection. This method can be extended to policy evaluation, program selection, and consultation decision-making, all of which involve complex environments.

\section{Data Availability}

All data and models generated or used during the study appear in the article. Some or all codes generated or used during the study are available from the corresponding author upon request.

\section{Conflicts of Interest}

The authors declare that they have no conflicts of interest.

\section{Acknowledgments}

This research was funded by the "Hsue-Shen Tsien's Life and Thought Research" Organization of the Shanghai Jiao Tong University in 2019, and the National Natural Science Foundation of China (U1501253), and Research on the Formation Factors and Successful Models of Strategic Scientists.

\section{References}

[1] Q. Tian, X. Han, and Y. Lei, "The status quo and future research directions of innovative talent cultivation theory," Road to Success, vol. 25, pp. 1-3, 2016.

[2] Y. Qiu, "Construction of the evaluation system for international applied innovative talents," Journal of Ningbo University (Humanities Edition), vol. 6, pp. 76-79, 2007.

[3] X. Li, "Research on quality and ability evaluation of higher vocational students," Educational Research, vol. 34, no. 05, pp. 96-103, 2013.

[4] S. Nan, F. Meng, B. Jiang, and W. Li, "Research on the construction of scientific and technological talent evaluation system under the innovation driven strategy," Scientific research management, vol. 37, no. S1, pp. 602-606, 2016.
[5] H. Shang and S. Wang, "Research on enterprise talent ecosystem evaluation from the perspective of value chain," Scientific research management, vol. 38, no. 01, pp. 153-160, 2017.

[6] M. Jia, L. Zhang, J. Fu, and L. Zhang, "Evaluation of high-level scientific and technological talents based on competitive evaluation," Scientific and technological progress and countermeasures, vol. 34, no. 16, pp. 120-125, 2017.

[7] A. W. Labib, G. B. Williams, and O'Connor, "RF an intelligent maintenance model (system): an application of the analytic hierarchy process and a fuzzy rule-based controller," Journal of the Operational Research Society, no. 49, pp. 745-757, 1998.

[8] C. Metin and I. Deha Er, "Structuring fuzzy integrated multistages evaluation model on academicpersonnel recruitment in MET institutions," Expert Systems with Applications, no. 36, pp. 6918-6927, 2009.

[9] S. P. Lazarevic, "Personnel selection fuzzy model," International Transactions in Operational Research, vol. 8, pp. 89-105, 2001.

[10] A. Drigas, S. Kouremenos, S. Vrettaros, and J. D. Kouremenos, "An expert system for job matching of the unemployed," Expert Systems with Applications, vol. 26, pp. 217-224, 2004.

[11] L. Shengzhao, Ed., Research Progress of Man-Machine-Environment System EngineeringVol. 5, Ocean Press, Beijing, 2001.

[12] X. Qian, J. Yu, and R. Dai, "A new field of science-open complex giant system and its methodology," Journal of $\mathrm{Na}$ ture, no. 01, pp. 3-64, 1990.

[13] L. A. Zadeh, "The concept of a linguistic variable and its application to approximate reasoning-I," Information Sciences, vol. 8, no. 3, pp. 199-249, 1975.

[14] G. Ali, H. Alolaiyan, D. Pamučar, M. Asif, and N. Lateef, “A novel MADM framework under q-rung orthopair fuzzy bipolar soft sets," Mathematics, vol. 9, no. 17, p. 2163, 2021.

[15] G. Ali, G. Muhiuddin, A. Adeel, and M. Zain Ul Abidin, "Ranking effectiveness of COVID-19 tests using fuzzy bipolar soft expert sets," Mathematical Problems in Engineering, p. 19, 2021.

[16] Rodríguez, M. Rosa, L. Martínez et al., "Hesitant fuzzy linguistic term sets," IEEE Transactions on Fuzzy Systems, vol. 20, pp. 109-119, 2012.

[17] Z. Xu and X. Gou, "Novel basic operational laws for linguistic terms, hesitant fuzzy linguistic term sets and probabilistic linguistic term sets," Information Sciences, vol. 372, pp. 407427, 2016.

[18] Q. Pang, H. Wang, and Z. Xu, "Probabilistic linguistic term sets in multi-attribute group decision making," Information Sciences, vol. 369, pp. 128-143, 2016.

[19] L. Wang and H. Xue, "Integrated decision-making method for heterogeneous attributes based on probabilistic linguistic cross-entropy and priority relations," Entropy, vol. 22, no. 9, p. 1009, 2020.

[20] S. Long, "The role and status of man-machine-environment system engineers in contemporary society," Research Progress of Man-Machine-Environment System Engineering, Vol. 7, China System Engineering Society, , Beijing, China, 2005.

[21] Y. Dong and Q. Wu, "Analysis of the current situation and countermeasures of the construction of young talents in the capital," in Proceedings of the Beijing Federation of Social Sciences Conference, Beijing, China, September 2012.

[22] G. Fan, "On the quality characteristics of young talents," Science and Technology Information, no. 1, p. 212, 2010.

[23] G. Fang and X. Wang, "Analysis of quality model of innovative talents based on personality traits," Journal of Northeastern University, no. 3, pp. 106-108, 2007. 
[24] Y. Feng, Research on Innovative Talents, pp. 101-102, Southwest Jiaotong University Press, Chengdu, China, 2006.

[25] G. Lei, Analysis on the Equity of China's Health System, Wuhan University, Wuhan, China, 2016.

[26] Y. Li, Research on Chinese People's Health Risk Model and Risk Assessment Method, Fourth Military Medical University, Xi'an, China, 2011.

[27] Y. Shen, "Recognition of the essence of mental health and health and its standards," Journal of Guyuan Teachers College, vol. 26, no. 5, pp. 37-39, 2005.

[28] H. Liu, "Re-recognition of the concepts and standards of mental health and health," Psychology and Science, vol. 38, no. 24 , pp. $480-481,2001$.

[29] C. Bai, R. Zhang, L. Qian, and Y. Wu, "Comparisons of probabilistic linguistic term sets for multi-criteria decision making," Knowledge-Based Systems, vol. 119, no. 3, pp. 284-291, 2017.

[30] W. Lei and X. Huifeng, "Integrated decision-making method for heterogeneous attributes based on probabilistic linguistic cross-entropy and priority relations," Entropy, vol. 22, no. 9, p. 1009, 2020.

[31] M. Zhao, X. Y. Shen, Y. F. He et al., "Probabilistic entropy and cross-entropy measures for multiple criteria decision making," Systems Engineering-Theory \& Practice, vol. 38, no. 10, pp. 2679-2689, 2018.

[32] X. Gou, Z. Xu, and H. Liao, "Hesitant fuzzy linguistic entropy and cross-entropy measures and alternative queuing method for multiple criteria decision making," Information Sciences, vol. 388-389, pp. 225-246, 2017.

[33] J. Q. Wang and J. T. Wu, "Method for multi-criteria Decisionmaking with hesitant fuzzy linguistic based on outranking relation," Control and Decision, vol. 30, no. 5, pp. 887-891, 2015.

[34] Xu Ze-shui and Da Qing-li, "Possibility degree method for ranking interval numbers and its application," Journal of Systems Engineering, no. 01, pp. 67-70, 2003.

[35] L. H. MaoY. F. Yang et al., "Construction of evaluation system of entrepreneurship based on entropy method," Statistics \& Decisions, vol. 6, pp. 156-160, 2020. 